\title{
The virtual fields method applied to spalling tests on concrete
}

\author{
F. Pierron ${ }^{1, a}$ and P. Forquin ${ }^{2}$ \\ ${ }^{1}$ LMPF, Arts et Metiers ParisTech, Rue St Dominique, BP. 508, 51006 Châlons en Champagne, France \\ ${ }^{2}$ LEM3, University of Lorraine, Ile du Saulcy, 57045 Metz Cedex 01, France
}

\begin{abstract}
For one decade spalling techniques based on the use of a metallic Hopkinson bar put in contact with a concrete sample have been widely employed to characterize the dynamic tensile strength of concrete at strain-rates ranging from a few tens to two hundreds of $\mathrm{s}^{-1}$. However, the processing method mainly based on the use of the velocity profile measured on the rear free surface of the sample (Novikov formula) remains quite basic and an identification of the whole softening behaviour of the concrete is out of reach. In the present paper a new processing method is proposed based on the use of the Virtual Fields Method (VFM). First, a digital high speed camera is used to record the pictures of a grid glued on the specimen. Next, fullfield measurements are used to obtain the axial displacement field at the surface of the specimen. Finally, a specific virtual field has been defined in the VFM equation to use the acceleration map as an alternative 'load cell'. This method applied to three spalling tests allowed to identify Young's modulus during the test. It was shown that this modulus is constant during the initial compressive part of the test and decreases in the tensile part when micro-damage exists. It was also shown that in such a simple inertial test, it was possible to reconstruct average axial stress profiles using only the acceleration data. Then, it was possible to construct local stress-strain curves and derive a tensile strength value.
\end{abstract}

\section{Introduction}

Among the techniques used to characterize the dynamic tensile strength of concrete figures prominently the spalling technique. The principle of this technique is the generation of a dynamic tensile loading due to the reflection of a short compressive pulse on the free surface of the concrete sample. A short cylindrical projectile launched by a gas gun facility against a metallic (steel or aluminum alloy) Hopkinson bar is generally employed to generate the compressive pulse. It propagates through the Hopkinson bar towards the contact surface of the concrete sample. Due to the impedance mismatch between the metal of the bar and the concrete a part of the incident wave is reflected in the opposite direction (upstream) whereas the other part is transmitted into the sample. This technique allows reaching strain-rates ranging from a few tens of $\mathrm{s}^{-1}$ to about $150 \mathrm{~s}^{-1}$. The spalling technique was initiated on concrete samples by Landon and Quinney [1] using an explosive charge at the end of a cylindrical specimen. The authors noticed a dynamic failure composed of multiple fracture planes. The use of a single Hopkinson bar to perform spalling tests was pioneered by Klepaczko and Brara [2]. Several improvements of the spalling technique have been proposed by Erzar and Forquin [3]. The use of a smaller Hopkinson bar and optimized smooth-end projectiles allowed obtaining more homogeneous stress field in the sample as well as higher strain rates (up to $180 \mathrm{~s}^{-1}$ ). However, several important limitations should also be pointed out. First, despite the recent use of high speed imaging [3] the kinetics of damage in the concrete sample remain quite uncertain. Moreover, even if the processing method provides the data of one-dimensional wave speed and the spall strength (maximum ultimate stress), the softening behaviour (in terms of stress-strain

a e-mail: fabrice.pierron@ensam.fr curve) of the concrete remains unknown and a direct identification of a damage evolution law is out of reach. For these reasons, full-field deformation measurements provide promising possibilities to improve the processing of the spalling tests.

Within the last decade, there has been spectacular progress in the diffusion of full-field deformation techniques, mainly due to increased computing power and availability of inexpensive CCD sensors following the development of general public digital cameras. White light techniques such as Digital Image Correlation [4] or moiré/grid methods [6] are now being routinely used in many experimental applications. A way of using the full potential of such measurements in order to identify the constitutive behaviour of materials requires processing the data with some inverse technique. A number of such inverse methods have been proposed in the past and the reader is referred to the following review article for details [7]. Among such inverse methods, The Virtual Fields Method (VFM) [5] is a technique fully dedicated to the processing of full-field measurements. Compared to finite element model updating, its strength is the fact that it does not require iterative resolutions of forward problems hence its excellent computational efficiency.

In the present paper, a spalling experiment performed on a common concrete specimen has been captured with a digital ultra-high speed camera (Shimadzu HPV-1). Each sample was covered by a grid so a full-field measurement method was used for measuring the displacement field in the axial direction. The data processing and measurement performances are first introduced then the results in terms of strain and strain-rate histories are presented and finally, the last part of the paper is dedicated to the identification of the mechanical properties of the concrete sample from the experimental data by using the Virtual Fields Method (VFM). 


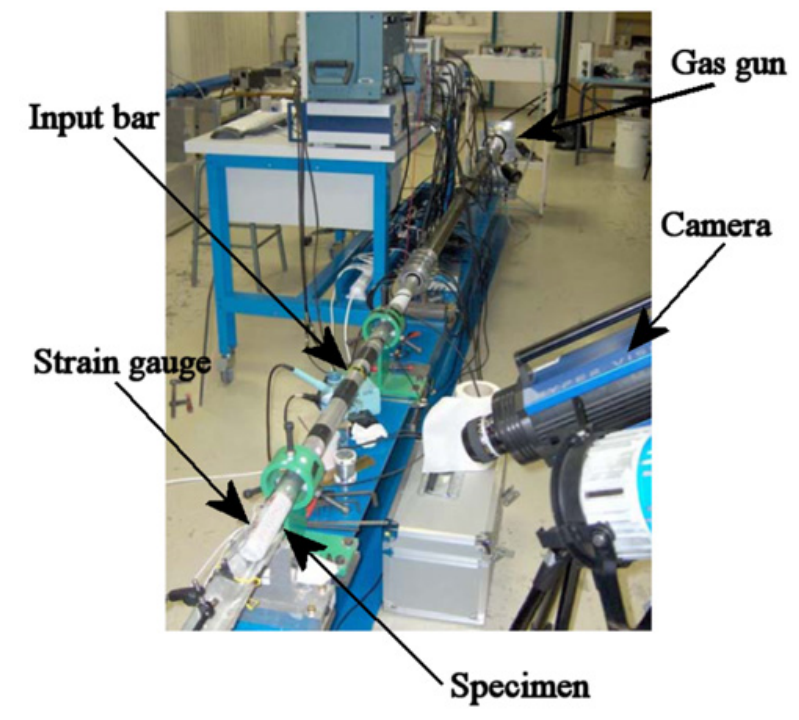

Fig. 1. Experimental set-up for the spalling tests.

\section{Experimental procedure}

The material tested in this study is a common concrete composed of hard siliceous aggregates with a maximum grain size of $8 \mathrm{~mm}$, sand, cement and water. The sample is cylindrical, $45.7 \mathrm{~mm}$ in diameter and $140 \mathrm{~mm}$ in length cored out from large concrete blocks. It has been ovendried at $60^{\circ} \mathrm{C}$ during several weeks until a stationary weight was reached (less than $0.1 \%$ loss of weight per day). The experimental set-up to perform the spalling tests is represented in Fig. 1. It is composed of a Hopkinson bar and spherical-cap ended projectile both made of the same high-strength aluminium alloy [3].

A Shimadzu HPV-1 ultra high speed camera was used to image the deformation of the specimen. This camera relies on a specific CCD sensor with onboard memory (also known as ISIS which stands for In-Situ Image Storage), with a spatial resolution of 312 by 260 pixels. It is beyond the scope of the present paper to give a complete description of the camera. Details can be found in [8]. An interframe time of $2 \mu$ s was used because the maximum available frame rate of $1 \mu$ s resulted in much worse images which were not suitable for small strain measurements. It was also necessary to rotate the camera so that the longitudinal axis of the specimen coincided with the transverse direction of the camera (the 260 pixels direction). This is because of the very low fill factor of the sensor in the other direction, creating unwanted moiré fringes. Finally, one needed to ensure that the images were dark, with grey level values below 100 to avoid spurious saturation effects. A details can be found in [8].

Displacement fields have been measured using an optical method called the grid method [6,9]. A grid pattern was deposited onto the surface of the specimen, consisting of a set of horizontal and vertical contrasted lines, with a natural spatial frequency $f_{0}$. Practically, these are obtained from a professional printer through an Encapsulated Postscript file. The prints consist of a polymer base and a photosensitive coating of very small thickness that provides the optical contrast. In order to avoid the presence of the thick polymer base at the surface of the specimen, the adopted procedure consists in bonding the film onto the specimen and then peeling off the base. A white glue is used to ensure good final contrast. Grids down to about 10 lines $\mathrm{mm}^{-1}$ can be obtained this way. For more details, the full procedure is reported in [10]. Images of the grid have been captured throughout the test. The first image is used as a reference. The spatial phase fields have been calculated using a windowed discrete Fourier transform [11]. The $u_{x}(x, y)$ and $u_{y}(x, y)$ displacement components relative to the unloaded configuration are calculated from the respective phase differences $\Delta \phi_{x}$ (for vertical lines) and $\Delta \phi_{y}$ (for horizontal lines) introduced by the deformation:

$$
\begin{aligned}
& u_{x}(x, y)=-\frac{p}{2 \pi} \Delta \phi_{x}(x, y) \\
& u_{y}(x, y)=-\frac{p}{2 \pi} \Delta \phi_{y}(x, y)
\end{aligned}
$$

where $p$ is the pitch of the grid. As it is well know for all fringe processing techniques, when the displacement becomes greater than the grid pitch here, phase wrapping occurs and phase unwrapping needs to be used. However, in the present case, the maximal displacement during the time of image acquisition and processing is lower than the grid pitch $(1 \mathrm{~mm})$ therefore no unwrapping was necessary.

Because of the high noise contents on the displacements and the fact that the strain values before damage are small (brittle material), significant low pass filtering is required to obtain meaningful strain values. This is at the cost of spatial resolution but it cannot be avoided here. The filtering procedure is a local fit of a second degree polynomial using a specific weighing function. This is referred to as diffuse approximation and details can be found in [12]. The strains are then obtained directly from the polynomial coefficients. The acceleration maps were obtained from the raw displacements by temporal fitting of a second order polynomial over a sliding window of 7 images. Again, the coefficients of the polynomials were used to derive the acceleration. Finally, the strain rate was obtained from the strain maps using a second order polynomial fit over a sliding window of 5 images. It is beyond the scope of the present paper to analyze in depth the performances of the Shimadzu HPV-1 camera in terms of deformation measurements. A thorough assessment of this camera can be found in [8]. The objective here is just to give the reader a feel for the measurement performances. The first twenty images when the wave has not reached the specimen yet were used to evaluate the performances. The displacement resolution is obtained as the standard deviation of the resulting displacement maps, using the first image as reference. Then, the processing reported above was used to produce strain, strain rate and acceleration. Again, the standard deviation for each of these quantities is reported in Table 1. A view of the specimen dimensions and the attached grid is given on.

\section{Stiffness identification}

In order to identify the material stiffness, the Virtual Fields Method (VFM) is used. This technique has been 
Table 1. Grid measurement information.

\begin{tabular}{|c|c|}
\hline Technique used & Grid method \\
\hline Pitch & $1 \mathrm{~mm}$ \\
\hline Sampling & 7 pixels per period $(\mathrm{N})$ \\
\hline Camera & $\begin{array}{l}\text { 8-bit } 312 \times 260 \text { Shimadzu HPV-1 } \\
\text { high speed camera }\end{array}$ \\
\hline Field of view & $24 \mathrm{~mm}$ by $37 \mathrm{~mm}$ \\
\hline Measurement points & 24 by 37 \\
\hline \multicolumn{2}{|l|}{ Displacement } \\
\hline Spatial resolution & $1.8 \mathrm{~mm}, 11$ pixels $(2 \mathrm{~N}-1)$ \\
\hline Resolution & $1.5 \mu \mathrm{m}, 0.01$ pixel, $\mathrm{p} / 650$ \\
\hline Temporal resolution & $2 \mu \mathrm{s}$ \\
\hline \multicolumn{2}{|l|}{ Strain } \\
\hline Smoothing method & Diffuse approximation, radius 8 \\
\hline Differentiation method & Analytical \\
\hline Spatial resolution & About $8 \mathrm{~mm}$ \\
\hline Resolution & $1.310^{-4}$ \\
\hline Temporal resolution & $2 \mu \mathrm{s}$ \\
\hline \multicolumn{2}{|l|}{ Strain rate } \\
\hline Smoothing method & $\begin{array}{l}2^{\text {nd }} \text { degree polynomial fitting along } \\
\text { time, sliding window of } 5 \text { data sets }\end{array}$ \\
\hline Differentiation method & Analytical \\
\hline Spatial resolution & About $8 \mathrm{~mm}$ \\
\hline Temporal resolution & $10 \mu \mathrm{s}$ \\
\hline Resolution & between 15 and $50 \mathrm{~s}^{-1}$ \\
\hline \multicolumn{2}{|l|}{ Acceleration } \\
\hline Smoothing method & $\begin{array}{l}2^{\text {nd }} \text { degree polynomial fitting along } \\
\text { time, sliding window of } 7 \text { data sets }\end{array}$ \\
\hline Differentiation method & Analytical \\
\hline Spatial resolution & $1.8 \mathrm{~mm}, 11$ pixels $(2 \mathrm{~N}-1)$ \\
\hline Temporal resolution & $14 \mu \mathrm{s}$ \\
\hline Resolution & $4.10^{4} \mathrm{~m} \cdot \mathrm{s}^{-2}$ \\
\hline
\end{tabular}

developed over the last twenty years to process full-field measurements with a view to identify material constitutive behaviour [5]. The idea relies on writing the principle of virtual work:

$$
-\int_{V} \sigma: \varepsilon^{\star} d V+\int_{\partial V} \mathbf{T} \cdot \mathbf{u}^{\star} d S+\int_{V} \mathbf{f} \cdot \mathbf{u}^{\star} d V=\int_{V} \rho \mathbf{a} \cdot \mathbf{u}^{\star} d V
$$

where $\sigma$ is the actual stress tensor, $\varepsilon^{\star}$ is the virtual strain tensor, $\mathbf{T}$ is the vector of surface distribution of applied loading acting on the solid boundary $\partial V, \mathbf{u}^{\star}$ is the virtual displacement vector, $\mathbf{f}$ is the vector of distribution of volume forces acting on the solid volume $V, \rho$ is the mass per unit volume, a the acceleration vector, '.' stands for the scalar product between two vectors and ':' stands for the contracted product between two second order tensors. Each time a virtual field is selected, Eq. (3) leads to an integral equilibrium equation that can be used to identify constitutive material parameters. In the present case, the volume forces can be neglected. One of the difficulties in high strain rate testing is the measurement of the impact force. This is the main reason why Hopkinson bars are used. In the present case, an innovative procedure is use. The idea is to define a virtual field that will zero out the contribution of $T$ in Eq. (3). In static elasticity, this leads to homogeneous linear equations that can only provide

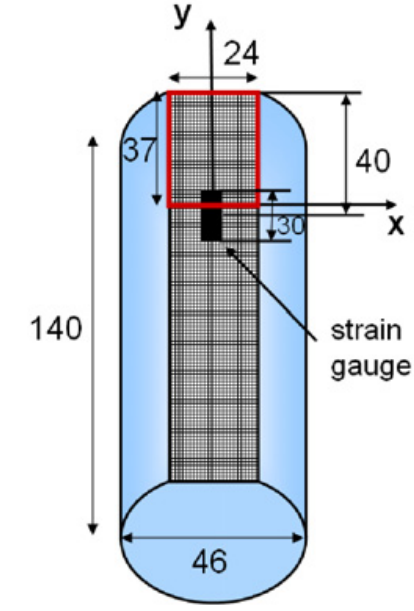

(a)

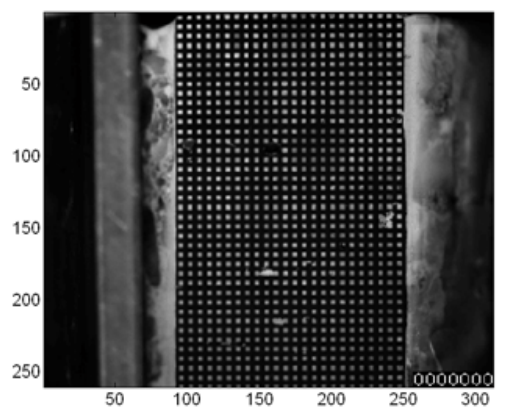

(b)

Fig. 2. Test specimen with field of view, dimensions and grid image. (a) Schematic of the specimen (dimensions in $\mathrm{mm}$ ); (b) Image of the field of view (red rectangle).

stiffness ratios (like Poisson's ratio). Indeed, without a force indication, absolute values of stiffnesses cannot be derived from kinematic measurements only. However, in dynamics, the right-hand side term of Eq. (3) can be used to an advantage. Indeed, in the instants of the tests when sufficient transient dynamic effects exist, then the knowledge of the material density coupled to the measurements of acceleration maps provide an alternative 'load cell', with the advantage that the information is embedded in the camera images. This is a very strong idea that has the potential to completely revolutionize dynamic testing in the future. It has already been validated for other high strain rate testing situations [13].

According to the test configuration as seen in Fig. 2, the following virtual field is defined:

$$
\left\{\begin{array} { l } 
{ u _ { x } ^ { * ( 1 ) } = 0 } \\
{ u _ { y } ^ { * ( 1 ) } = f ( y ) }
\end{array} \left\{\begin{array}{l}
\varepsilon_{x}^{*(1)}=0 \\
\varepsilon_{y}^{*(1)}=f^{\prime}(y) \\
\varepsilon_{s}^{*(1)}=0
\end{array}\right.\right.
$$

where $f$ is a continuous and differentiable function of $y$, with $\mathrm{f}(0)=0$ so as to cancel out the contribution of the stress at the bottom boundary of the field of view (see Fig. 2). Assuming that the stress state is uniaxial, one can 
write $\sigma_{y}=E \varepsilon_{y}$ and the principle of virtual work becomes

$$
-E \int_{V} \varepsilon_{y} f^{\prime}(y) d V=\rho \int_{V} a_{y} f(y) d V
$$

The assumption that $\varepsilon_{y}$ and $a_{y}$ only depend on $y$ means that the volume integrals can be transformed into line integrals

$$
-E \int_{0}^{L} \varepsilon_{y} f^{\prime}(y) d y=\rho \int_{0}^{L} a_{y} f(y) d y
$$

where $L$ is the length of the field of view. Finally, the continuous integrals can be approximated by discrete sums as is usual in the VFM

$$
-E \int_{0}^{L} \varepsilon_{y} f^{\prime}(y) d y \approx-E \frac{L}{n_{1}} \sum_{i=1}^{n_{1} n_{2}} \varepsilon_{y}^{i} f^{\prime}\left(y^{i}\right)
$$

and

$$
\rho \int_{0}^{L} a_{y} f(y) d y \approx \rho \frac{L}{n_{1}} \sum_{i=1}^{n_{1} n_{2}} a_{y}^{i} f\left(y^{i}\right)
$$

where $n_{1}$ and $n_{2}$ are the number of measurement points in the $x$ and $y$ directions respectively (see Table 1). Dividing these equations by $n_{2}$ and denoting

$$
\overline{\varepsilon_{y} f^{\prime}(y)}=\frac{1}{n_{1} n_{2}} \sum_{i=1}^{n_{1} n_{2}} \varepsilon_{y}^{i} f^{\prime}\left(y^{i}\right)
$$

and

$$
\overline{a_{y} f(y)}=\frac{1}{n_{1} n_{2}} \sum_{i=1}^{n_{1} n_{2}} a_{y}^{i} f\left(y^{i}\right)
$$

Young's modulus is given by

$$
E=-\rho \frac{\overline{a_{y} f(y)}}{\overline{\varepsilon_{y} f^{\prime}(y)}}
$$

This is a very simple expression that provides a value for $E$ at each time step of the test. The only problem that remains is the selection of the virtual field. For this purpose, one criterion to choose $f$ is that it maximizes both terms of the fraction in Eq. (11). One way to ensure this in an easy manner is to define $f$ using the actual measurements. To do so, at each time $t$ when an image is recorded, the $u_{y}$ displacement map is averaged over $x$, leading to a vector depending only on $y$. It is then smoothed using a $6^{\text {th }}$ degree polynomial and expanded back in the $x$ direction to provide a $2 \mathrm{D}$ virtual field that only depends on $y$, as required by Eq. (11). The reason for this smoothing is to provide extra filtering in the calculation of the acceleration term because spatial noise is still significant there. Finally, a constant value is added so that the virtual displacement is zero at $y=0$.

Figure 3 shows the time history of the two terms in the fraction of Eq. (11). First, the term associated to the virtual work of internal forces $\overline{\varepsilon_{y} f^{\prime}(y)}$ is shown in Fig. 3(a). The values before $40 \mu$ s enable to define a noise threshold. Below this value, the data are considered not significant. Moreover, if the data are above but close to this threshold, the influence of noise will be important and the identified Young's modulus will not be accurate. It was decided here

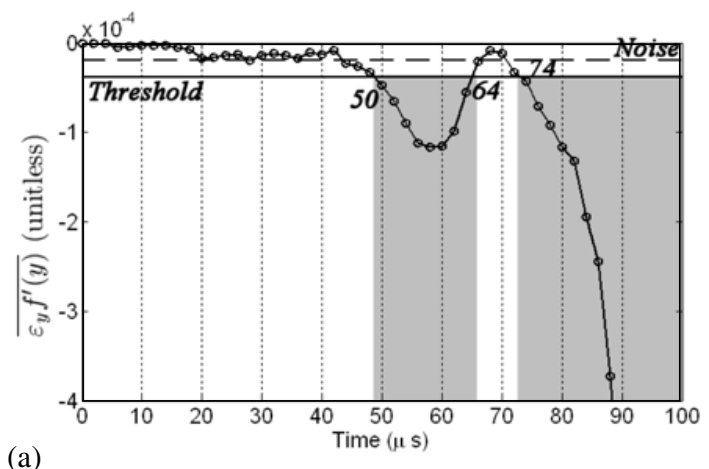

(a)

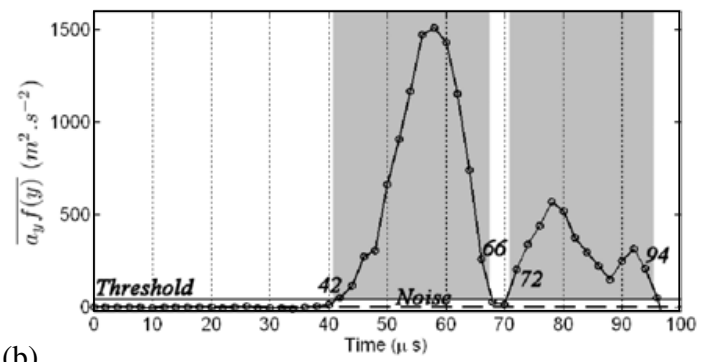

(b)

Fig. 3. Time history of the two terms in Eq. (11). (a) $\overline{\varepsilon_{y} f^{\prime}(y)}$ (unitless); (b) $\overline{a_{y} f(y)}\left(m^{2} \cdot s^{-2}\right)$ (unitless).

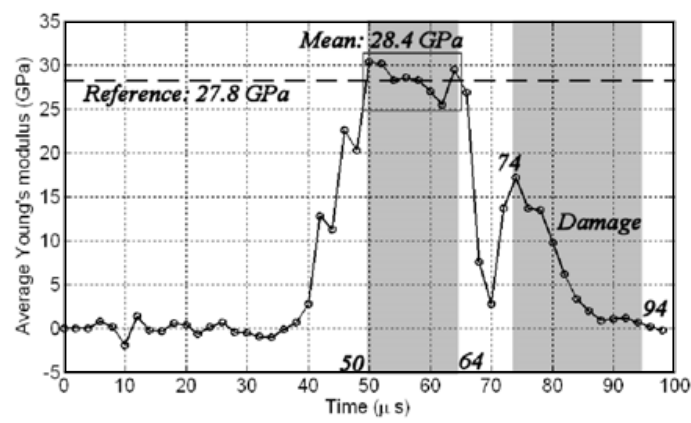

Fig. 4. Identified Young's modulus history.

to take a threshold of twice the noise level, as reported on the plot. One can then specify the intervals when the identification will be possible. For $\overline{\varepsilon_{y} f^{\prime}(y)}$, a first interval between 50 and $64 \mu \mathrm{s}$ is defined, corresponding to the compression stage of the test. Then, the strains become very small during the reflection of the wave, and they pick up again after $74 \mu$ s with a spectacular increase after $80 \mu \mathrm{s}$ corresponding to the onset of the macrocrack. The second term, $a_{y} f(y)$, is shown in Fig. 3(b). Again, a noise limit is identified and a threshold defined as twice this limit. Intervals of sufficiently significant values are then defined as previously, with a first interval between 42 and $66 \mu \mathrm{s}$, and a second between 72 and $94 \mu$ s. Interestingly, these two plots clearly show that the noise of the strain term is the predominant one, which conditions the intervals over which $E$ can be expected to be identified correctly. Finally, Fig. 4 shows the values of $E$ identified along the test. On this plot, the intervals of significance have been reported using the intersection of the intervals from Fig. 3.

The results are rather spectacular considering the very small strain levels involved. During the compressive stage of the test, between 50 and $64 \mu$ s when strain data are 
significant, a rather constant value of $E$ is identified. If the values are averaged over the 8 load steps in this interval, an average value of $28.4 \mathrm{GPa}$ is obtained. The reference value of $27.8 \mathrm{GPa}$ reported on the plot comes from the measurement of the wave speed $C_{0}$ using the three strain gauges positioned along the specimen, as described in [3]. A value of $3500 \mathrm{~m} . \mathrm{s}^{-1}$ is obtained for $C_{0}$ while weighting of the specimen yielded a density $\rho$ of $2271 \mathrm{~kg} \cdot \mathrm{m}^{-3}$. $E$ was then obtained by $E=\rho C_{0}^{2}$. The two estimates of Young's modulus are very close, which confirms the validity of the present identification approach as well as the quantitative nature of the kinematic information (strain, acceleration). Looking at the second significance interval, between 74 and $94 \mu$ s when the wave is tensile, the Young's modulus values are much lower and a steady decrease is observed from $17 \mathrm{GPa}$ at $74 \mu \mathrm{s}$ down to nearly zero at $94 \mu \mathrm{s}$. This is believed to be caused by the damage progress in tension that ultimately leads to macrocracking.

\section{Stress reconstruction}

The objective of this section is to present an alternative data processing procedure taking advantage of the inertial nature of the test. Indeed, considering the field of view of the specimen and taking now the reference of the coordinate system at the specimen free end, one can define the following virtual field (rigid body like virtual field):

$$
\left\{\begin{array} { l } 
{ u _ { x } ^ { * } = 0 } \\
{ u _ { y } ^ { * } = 1 }
\end{array} \left\{\begin{array}{l}
\varepsilon_{x}^{*(1)}=0 \\
\varepsilon_{y}^{*(1)}=0 \\
\varepsilon_{s}^{*(1)}=0
\end{array}\right.\right.
$$

where $b(y)$ is the length of the considered specimen portion measured from the free edge and $S$ the cross-section. Denoting $\overline{\sigma_{y}(y, t)}$ the average of the axial stress in transverse sections (average over $x$ ), the different terms in the principle of virtual work become:

$$
\begin{aligned}
-\int_{V} \sigma: \varepsilon^{\star} d V & =0 \\
\int_{\partial V} \mathbf{T} \cdot \mathbf{u}^{\star} d S & =S \overline{\sigma_{y}(y, t)} \\
\int_{V} \rho \mathbf{a} \cdot \mathbf{u}^{\star} d V & =-S \rho b(y) \overline{a_{y}(y, t)}
\end{aligned}
$$

Finally, the $\overline{\sigma_{y}(y, t)}$ stress can be reconstructed at any section of coordinate $y$ with $y$ between 0 and L by:

$$
\overline{\sigma_{y}(y, t)}=-\rho b(y) \overline{a_{y}(y, t)}
$$

It should be noted that the minus sign in front of the right-hand side term comes from the fact that the acceleration has been defined in the coordinate axes of Fig. 2 . Therefore, $\overline{a_{y}(y, t)}$ is positive during the compression wave. So, the minus sign does in fact give $\overline{\sigma_{y}(y, t)}$ negative, as expected for a compression wave. It is interesting to note that this equation is independent from the material constitutive behaviour, underlining the fact that the virtual work of the inertial forces act as a load cell. It is also worth mentioning that the same idea was already proposed in [14] but writing the equilibrium of sections without the

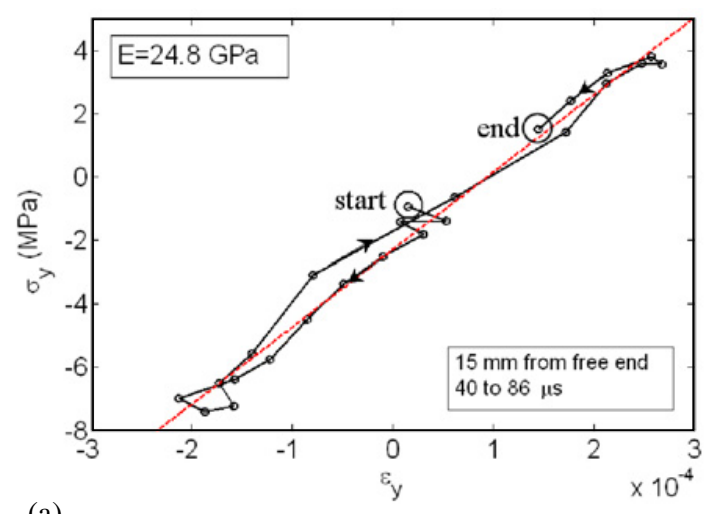

(a)

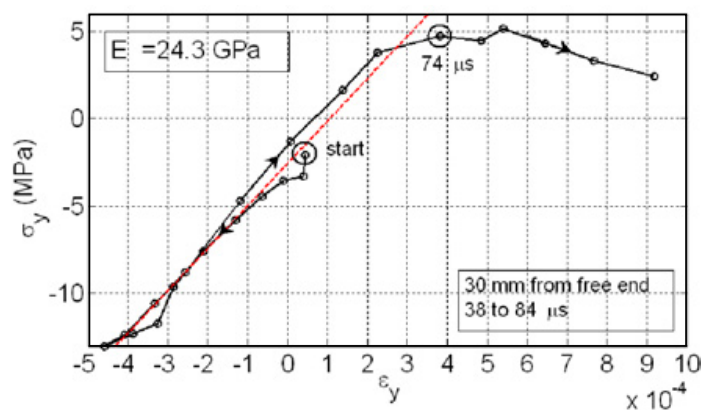

(b)

Fig. 5. Stress-strain curves, red line indicates linear regression on linear elastic portion of the test. (a) $15 \mathrm{~mm}$ from free end; (b) $30 \mathrm{~mm}$ from free end.

use of the integral form of equilibrium. The authors called this a "non-parametric" method because the equation does not depend on the material constitutive parameters. Now that stress profiles can be reconstructed at any time and any transverse section, it is possible to draw stress-strain curves for different sections. Figure 5 shows the stressstrain curves for two sections, one where there is no damage ( $15 \mathrm{~mm}$ from the free end) and one where there is significant damage ( $30 \mathrm{~mm}$ from the free end). It is clear from Fig. 5(a) that the response is linear elastic until the end of the video capture (and presumably until the end of the test since after $86 \mu \mathrm{s}$, damage has already localized and the stress waves are dying out). On the other hand, on Fig. 5(a), significant damage occurs after $74 \mu$ s and the stress-strain curve flattens out. Using all the sections data, it may be possible to identify a damage law. However, the spatial resolution of the measurements is too poor to have a detailed account of the damage evolution for the moment. Again, the future progress of ultra high speed cameras should enable to bring the present analysis one step further.

\section{Conclusion and future work}

This paper has presented the results obtained on a spalling experiment performed on a concrete cylindrical rod. Fullfield strain measurements were obtained through images of grids captured by an ultra high speed camera (Shimadzu HPV-1). The main conclusions are as follows.

- The grid method was successfully used to measure fullfield displacements on the concrete specimen. A strain 
resolution of about $1.510^{-4}$ was obtained for a spatial resolution of about 50 pixels. Such performances would not have been possible with digital image correlation. It was obtained at the cost of bonding a grid at the surface of the specimen.

- From these measurements, the Virtual Fields Method was used to identify an average value of Young's modulus of the material by assuming that the stress state was uniaxial. The acceleration forces were used as an embedded load cell to perform the identification, leading to a value of Young's modulus at each time when a frame is captured.

- It was shown that during the first part of the test, when the input compressive wave travels along the specimen, the modulus is constant and close to the reference value obtained by the wave speed measured through strain gauges. Then, in the tensile part of the test, the average Young's modulus is found to decrease with time, a witness of the damage process taking place in the specimen before the onset of macroscopic cracking.

- Finally, it was shown that for such a simple inertial test, it was possible to reconstruct average longitudinal stress profiles using only the acceleration data. Then, it is possible to construct local stress-strain curves and derive a tensile strength value. Although the temporal and strain resolutions are just not quite good enough to identify a local damage law, there is not much missing to be able to do this in the near future when camera performances increase. In fact, it would be interesting to repeat these tests with the Cordin 550-62 used in [13] with a frame rate of $0.5 \mu \mathrm{s}$.

- The present study is exploratory in nature and aims at the long-term goal of developing novel high strain rate testing approaches relying on full-field measurements and inverse identification. The idea of using the acceleration maps in the process is of particular interest for future work and confirms other recent results based on the same approach [13].

\section{Acknowledgements}

This work was performed under a program entitled 'Experimental approach and modelling of the dynamic behaviour of brittle materials (Exam-Brittles)' sponsored by the French National Agency for Research (ANR) and supported by the CEA, DAM, GRAMAT. These supports are gratefully acknowledged. The authors would like to thank Dr Benjamin Ezrar for helping out with the experiments, as well as Dr Marco Rossi for useful discussions about the Shimadzu camera.

\section{References}

1. J.W. Landon, H. Quinney, Proc. of the Royal Soc., 103(723), (1923) 622-643

2. J.R. Klepaczko, A. Brara, An experimental method for dynamic tensile testing of concrete by spalling, Int. J. of Impact Eng., 25, (2001) 387-409

3. B. Erzar, P. Forquin, An experimental method to determine the tensile strength of concrete at high rates of strain, Exp. Mech., 50, (2010) 941-955

4. M.A. Sutton, J.-J. Orteu, Schreier H., Image correlation for shape, motion and deformation measurements (Springer, New-York 2009)

5. F. Pierron and M. Grédiac, The Virtual Fields Method (Springer, New-York, 2012)

6. Y. Surrel, Interferometry ' 94 : Photomechanics, SPIE 2342, (1994)

7. S. Avril, M. Bonnet, A.S. Bretelle, M. Grédiac, F. Hild, P. Ienny, F. Latourte, D. Lemosse, S. Pagano, S. Pagnacco, F. Pierron, Exp. Mech., 48(4), (2008) 381-402

8. M. Rossi, R. Cheriguene, F. Pierron, P. Forquin, Proc. of 2011 SEM Annual Conference and Exposition on Experimental and Applied Mechanics, 13-16 June 2011 in Mohegan Sun, CT, USA

9. S. Avril, E. Ferrier, P. Hamelin, Y. Surrel, A. Vautrin, Comp. Part A, 35(7-8), (2004) 873-884

10. J.-L. Piro, M. Grédiac, Exp. Tech., 28, (2004) 23-26

11. Y. Surrel, Applied Optics, 35, (1996) 61-60

12. S. Avril, P. Feissel, F. Pierron, P. Villon, Meas. Sci. and Tech., 17, (2008) 857-868

13. R. Moulart, F. Pierron, S.R. Hallett, M.R. Wisnom, Exp. Mech., 51(4), (2011) 509-536

14. R. Othman, S. Alaoui, A. Poitou, Polymer Testing, 29(5), (2010) 616-623 